\title{
Serum pregnancy-associated plasma protein A (PAPPA) as a predictor of eosinophilic Type-2 high asthma
}

Kian Fan Chung ${ }^{1}$, qingling Zhang ${ }^{2}$, Jiaxing Xie ${ }^{1}$, Ian Adcock ${ }^{1}$, Nazanin Kermani ${ }^{3}$, Stelios Pavlidis $^{3}$, Kai Sun ${ }^{3}$, Yike Guo ${ }^{3}$, Peter Sterk ${ }^{4}$, Sven-Erik Dahlén ${ }^{5}$, Richard Knowles G6 ${ }^{6}$, and Ratko Djukanovic $^{7}$

${ }^{1}$ Imperial College London National Heart and Lung Institute

${ }^{2}$ Guangzhou Medical College First Affiliated Hospital

${ }^{3}$ Imperial College London

${ }^{4}$ Amsterdam Universitair Medische Centra

${ }^{5}$ Karolinska Universitetssjukhuset Medicinsk Teknik

${ }^{6}$ Stevenage Bioscience Catalyst

${ }^{7}$ University of Southampton

July 9, 2021

\section{Serum pregnancy-associated plasma protein A (PAPPA) as a predictor of eosinophilic Type-2 high asthma}

To the Editor,

Pregnancy-associated plasma protein A (PAPPA), a metalloproteinase that cleaves insulin-like growth factor (IGF)-binding proteins (IGFBPs) to increase IGF availability, is expressed systemically in pregnant women but also in other tissues (1). Higher serum PAPPA levels are reported in patients with newly-diagnosed asthma (1) and allergic rhinitis compared to healthy controls and are decreased following omalizumab treatment (2). We determined whether PAPPA could represent a novel biomarker for Type-2 (T2) asthma by exploring the relationship between asthma severity and phenotypes of severe asthma and PAPPA gene and protein expression (3).

We recruited 288 severe non-smoking asthma (NSA), 102 smokers and ex-smokers with severe asthma (SSA), 86 mild/moderate non-smoking asthmatics (MMA) and 95 healthy non-smoking controls (HC) from the U-BIOPRED cohort (NCT01976767) (4) (Table S1 ). Transcriptomic and proteomic profiling of blood and sputum samples and specific serum periostin ELISA were performed (3). Gene set variation analysis (GSVA) was used to calculate the enrichment score (ES) of 34 genes that were upregulated following in vitro stimulation of primary human bronchial epithelial cells with IL-13 (T2_IL-13_IVS) (3). Eosinophilic inflammation was defined by sputum eosinophilia $>1.49 \%$ (3). Local Ethics Committees of the recruiting centres approved the study and all participants gave written informed consent.

Sputum cell PAPPA mRNA was elevated in NSA compared to SSA, MMA and HC subjects particularly in granulocytic asthmatics and in the transcriptomic-associated cluster (TAC)1; an eosinophilic cluster (5)

(Figure 1A-C ). This was more pronounced with sputum PAPPA protein analysis according to asthma severity, in eosinophilic and mixed granulocytic asthmatics and in T2-high asthmatics identified by the T2_IL-13_IVS signature (Figure 1D-F ).

PAPPA mRNA expression in blood cells was similar across asthma severities, blood granulocytes and molecular phenotypes (Supplementary Figure 1A-C ). However, serum PAPPA protein levels supported the 
discrimination seen in sputum with significant elevation seen in SA compared to HC, in eosinophilic and mixed granulocytic asthma and in T2-high asthma (SupplementaryFigure 1D-F ).

Sputum eosinophil percentages were significantly correlated with sputum $\left(\mathrm{r}=0.88, \mathrm{p}=10^{-6}\right)$ and serum $\left(\mathrm{r}=0.41, \mathrm{p}=10^{-6}\right)$ PAPPA protein levels. Overall, sputum PAPPA protein gave a greater distinction between asthma severity, granulocyte composition and T2-high asthma than with serum although fewer samples were available.

These results were validated in sputum from the Airways Disease Endotyping for Personalized Therapeutics (ADEPT) study (6) (Supplementary Figure S2 ). Elevated PAPPA protein in the serum and sputum of severe asthmatics and in eosinophilic compared to non- eosinophilic subjects was seen (SupplementaryFigure S2A-D ). In addition, sputum PAPPA mRNA levels were also elevated in eosinophilic versus non-eosinophilic asthma in the ADEPT cohort (Supplementary Figure S2E ).

The ES score of the T2 IL-13_IVS gene signature in bronchial brushings was significantly, but weakly, correlated with blood eosinophil counts $\left(\mathrm{r}=0.329, \mathrm{p}=10^{-6}\right)$, serum PAPPA $\left(\mathrm{r}=0.356, \mathrm{p}=10^{-6}\right)$, but not with serum periostin levels $(\mathrm{r}=0.07$, $\mathrm{p}$-value $=0.48)$. In contrast, the T2 IL-13 IVS ES score was strongly correlated with sputum PAPPA levels $\left(\mathrm{r}=0.72, \mathrm{p}=10^{-3}\right)$. Sputum PAPPA protein levels also significantly correlated with markers of remodelling such as MMP10 $\left(\mathrm{r}=0.646, \mathrm{p}<10^{-6}\right)$ and $\operatorname{MET}\left(\mathrm{r}=0.429, \mathrm{p}<10^{-6}\right)$.

Receiver-operating characteristics (ROC) curve analysis was performed for sputum eosinophilia (Supplementary Table S2). The area under the ROC curve (AUC) for serum indicated that there was no good predictor although blood eosinophilia was the best (0.79) being marginally better than serum PAPPA and exhaled NO (Figure 2A ). In contrast, sputum PAPPA was an excellent predictor of sputum eosinophilia (0.98), better than blood eosinophilia and exhaled nitric oxide levels (Figure 2B ).

Therefore, sputum PAPPA is an excellent biomarker for sputum eosinophilia and for T2-high asthma whilst serum PAPPA is as effective as blood eosinophilia in predicting high sputum eosinophil levels and with T2-high asthma.

\section{Legend to Figures}

Figure 1. Dot and box plot (median and 95\% CI) of sputum cell PAPPA mRNA expression scores in: (A) never-smoking severe asthma (NSA), smoking and ex-smoking SA (SSA), mild-moderate asthma (MMA) and healthy controls (HC); (B) in eosinophilic, neutrophilic, mixed and pauci-granulocytic inflammation in asthmatics and healthy controls (HC); and (C) in the transcriptomic-associated clusters (TAC1, TAC2 and TAC3) in U-BIOPRED. Sputum PAPPA protein levels in NSA, SSA, MMA and HC subjects (D); eosinophilic, neutrophilic, mixed and pauci airway inflammation subjects and HC (E); in T2-high and T2low asthmatic subjects and HC subjects (F).

Figure 2. Receiver operating characteristic (ROC) curves analysis of the sensitivity and specificity of serum (A) and sputum (B) PAPPA levels for sputum eosinophilic airway inflammation (sputum eosinophils $>1.49 \%$ ) in asthmatic subjects. AUC, Area under the curve.

Jiaxing Xie ${ }^{1}$, Nazanin Zounemat Kermani ${ }^{1,2}$, Stelios Pavlidis ${ }^{1,2}$, Kai Sun ${ }^{2}$, Yike Guo ${ }^{2}$, Richard G Knowles ${ }^{3}$, Qingling Zhang ${ }^{4}$, Sven-Erik Dahlen ${ }^{5}$, Peter Sterk ${ }^{6}$, Ratko Djukanovic ${ }^{7}$, Kian Fan Chung ${ }^{1,2}$, Ian M. Adcock ${ }^{1,2}$ on behalf of the U-BIOPRED study group \#

${ }^{1}$ National Heart and Lung Institute, Imperial College London, and Biomedical Research Unit, Royal Brompton and Harefield NHS Trust, London, UK; ${ }^{2}$ Dept of Computing and Data Science Institute, Imperial College London, London, UK; ${ }^{3}$ Knowles Consulting, Stevenage Bioscience Catalyst, Stevenage, UK; ${ }^{4}$ Pulmonary and Critical Care Medicine, Guangzhou Institute of Respiratory Health \& The First Affiliated Hospital of Guangzhou Medical University, Guangzhou 510120, China; ${ }^{5}$ Centre for Allergy Research, Karolinska Institute, Stockholm, Sweden; ${ }^{6}$ Amsterdam University Medical Centers, University of Amsterdam, Amsterdam, Netherlands $;{ }^{7}$ NIHR Southampton Respiratory Biomedical Research Unit, University Hospital Southampton, Southampton, UK . 
\# The list of U-BIOPRED study group members is provided in an online supplementary Table.

\section{Hosted file}

image1.wmf available at https://authorea.com/users/322383/articles/529699-serum-pregnancyassociated-plasma-protein-a-pappa-as-a-predictor-of-eosinophilic-type-2-high-asthma

Figure 1

\section{Hosted file}

image2.wmf available at https://authorea.com/users/322383/articles/529699-serum-pregnancyassociated-plasma-protein-a-pappa-as-a-predictor-of-eosinophilic-type-2-high-asthma

\section{Figure 2}

\section{References:}

1. Coskun A, Balbay O, Duran S, Annakkaya AN, Bulut I, Yavuz O, et al. Pregnancy-associated plasma protein-A and asthma. Adv Ther. 2007;24(2):362-7.

2. Bulut I, Ozseker ZF, Coskun A, Serteser M, Unsal I. Pregnancy-associated plasma protein-A (PAPP-A) levels in patients with severe allergic asthma are reduced by omalizumab. J Asthma. 2018;55(10):111621.

3. Pavlidis S, Takahashi K, Ng Kee Kwong F, Xie J, Hoda U, Sun K, et al. "T2-high" in severe asthma related to blood eosinophil, exhaled nitric oxide and serum periostin. Eur Respir J. 2019;53(1).

4. Shaw DE, Sousa AR, Fowler SJ, Fleming LJ, Roberts G, Corfield J, et al. Clinical and inflammatory characteristics of the European U-BIOPRED adult severe asthma cohort. Eur Respir J. 2015;46(5):1308-21.

5. Kuo CS, Pavlidis S, Loza M, Baribaud F, Rowe A, Pandis I, et al. A Transcriptome-driven Analysis of Epithelial Brushings and Bronchial Biopsies to Define Asthma Phenotypes in U-BIOPRED. Am J Respir Crit Care Med. 2017;195(4):443-55.

6. Silkoff PE, Strambu I, Laviolette M, Singh D, FitzGerald JM, Lam S, et al. Asthma characteristics and biomarkers from the Airways Disease Endotyping for Personalized Therapeutics (ADEPT) longitudinal profiling study. Respiratory research. 2015;16:142. 
figures/Figure-1/Figure-1-eps-converted-to.pdf 
figures/Fig-2/Fig-2-eps-converted-to.pdf 